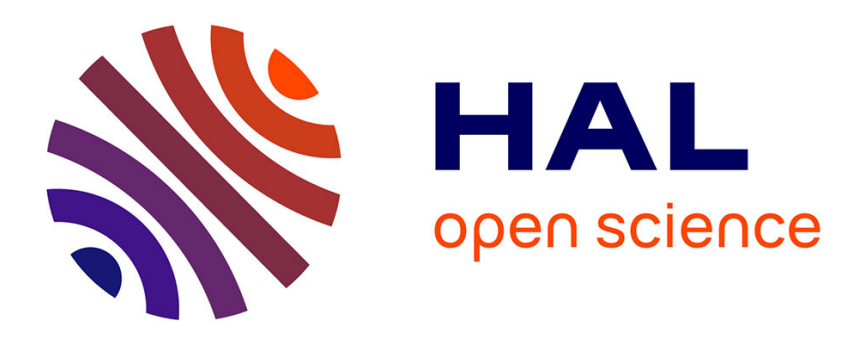

\title{
Dénommer, désigner en classe: aspects du métalangage et interactions
}

Catherine Boré

\section{To cite this version:}

Catherine Boré. Dénommer, désigner en classe: aspects du métalangage et interactions. Dénommer, désigner en classe: aspects du métalangage et interactions, Feb 2007, France. halshs-00355876

\section{HAL Id: halshs-00355876 \\ https://shs.hal.science/halshs-00355876}

Submitted on 26 Jan 2009

HAL is a multi-disciplinary open access archive for the deposit and dissemination of scientific research documents, whether they are published or not. The documents may come from teaching and research institutions in France or abroad, or from public or private research centers.
L'archive ouverte pluridisciplinaire HAL, est destinée au dépôt et à la diffusion de documents scientifiques de niveau recherche, publiés ou non, émanant des établissements d'enseignement et de recherche français ou étrangers, des laboratoires publics ou privés. 
Catherine Boré

MCF Sciences du langage

UCP- IUFM Versailles

Modyco UMR 7114 CNRS-Université Paris X

\section{Dénommer, désigner en classe : aspects du métalangage et interactions}

\section{Résumé :}

A partir de transcriptions d'oraux de fin d'école primaire recueillis dans la même classe en géométrie et en grammaire, sont repérées deux conduites discursives récurrentes à l'école : dénommer et désigner, conduites qui impliquent la production et la compréhension de définitions et leur mise en circulation dans le langage. En analysant l'autonymie dans les interactions, on met en évidence :

- sa puissance de thématisation dans les échanges inter-élèves et maître /élèves

- son usage opposé dans deux disciplines différentes

- sa capacité de révélateur des conflits cognitifs.

Mots-clés : autonymie, rédaction conversationnelle, mot métalinguistique, interaction, désigner, dénommer.

\section{INTRODUCTION}

Avec la nomination, s’offrent deux cas de figure opposés, dénommer et désigner, qui constituent traditionnellement une alternative entre deux points de vue distincts : la définition qu'en donnent Siblot et al. $\left(^{1}\right)$ va dans ce sens très général qui définit deux types d’opérations sémantiques. Josette Rey-Debove, quant à elle, traite expressément de l'autonymie, et part des

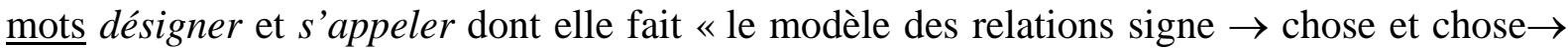
signe, c'est-à-dire la mise en relation du monde et des signes en passant du système lexical mondain au système métalinguistique » $\left({ }^{2}\right)$. J'ai privilégié cette analyse, traduite par deux formulations sémiotiques opposées qui sont présentées un peu plus loin. Je dispose ainsi d’un modèle de phrase métalinguistique qui me permettra d'analyser quelques exemples sélectionnés dans un corpus oral recueilli en classe.

La classe est tout particulièrement, en effet, le lieu de la nomination, et l'étiquetage des notions constitue une part importante des apprentissages. Dans certaines disciplines comme la

\footnotetext{
${ }^{1}$ «Dénommer, c'est donner le nom d'une catégorie, catégoriser un référent en l'insérant dans une classe d'objets identifiés dans le lexique. Tandis que désigner renvoie à un élément du monde dont on désigne un aspect contingent. » SIBLOT, Paul (dir.) \& al., Termes et concepts pour l'analyse du discours, une approche praxématique, Champion, Paris, 2001, p.205.

${ }^{2}$ REY-DEBOVE, Josette, Le Métalangage, Paris, Le Robert, 1978, p.185. Voir aussi p.26 (je résume) : un ensemble de mots «mondains » est employé pour parler de tout ce qui n'est pas le langage mais le monde ou les choses (ex : feu, soleil) ; un ensemble de mots «métalinguistiques» est employé pour parler du langage (ex : mot, phrase, illisible, dire, etc.).
} 
Catherine Boré UCP-IUFM Versailles et Modyco UMR 7114 CNRS-Université Paris X Journée InterGAP «Interactions et apprentissages » 9 février 2007

géométrie ou la grammaire, on ne peut se passer de métalangage spécialisé. Celui-ci s’ajoute et s'enchevêtre parfois avec le métadiscours qui lui sert de cadre. La manière dont il est introduit, l'usage qui est fait de l'autonymie, constituent souvent un style didactique: le moment et la manière avec laquelle on introduit le nom d'une notion, la forme donnée à une définition conditionnent les apprentissages.

La communication qui suit se situe dans le cadre général d'une didactique des apprentissages ${ }^{3}$ qui met en oeuvre la dynamique d'une co-construction des savoirs maîtreélèves et élèves-élèves. Les références sont empruntées principalement aux domaines de la linguistique de l'énonciation, de l'analyse de discours, avec une référence au champ de la psychologie sociale. Dans le cadre d'une co-construction des savoirs, l'activité définitoire est partagée par l'enseignant et les élèves.

Elle prend des formes diverses, en fonction des types d'interactions maître- élèves et élèves élèves, et en fonction des disciplines.

Les différences portent :

-sur l'objet visé par la nomination : dénommer une phrase occurrente complétive ou relative et donner le nom des côtés (opposé, adjacent) d'un triangle rectangle tracé au tableau, est-ce la même activité sémiotico-cognitive ? Ce qui est visé par la dénomination n’a pas exactement le même statut selon qu'il s'agit de la pré-construction d'un référent, d'une notion à construire, qui passe par l'identification d'un token, ou quand il s'agit de l'objet «angle droit », par exemple, déjà schématisé.

-sur l’objet visé par la désignation : il peut s'agir tout autant de donner le sens des mots perpendiculaire ou proposition, que consigne, c'est-à-dire, dans ce dernier cas, des mots qui servent à énoncer le discours. Ce sont des strates différentes du discours didactique qui se trouvent présentes à la fois.

- sur l'activité des élèves, à l'intérieur des deux disciplines : dans les exemples choisis, une part de l'activité va consister à faire la synthèse orale des notions qui ont été construites

\footnotetext{
${ }^{3}$ Elle s'appuie sur les conclusions d'une recherche menée dans le cadre d'un appel d'offres de l'IUFM de Versailles centré sur la relation entre les pratiques d'enseignement et leurs effets sur les élèves (1998-2000, 2000-2003). Cette recherche, qui associait données orales et écrites dans l'apprentissage de la production d'écrits, a donné lieu à une publication en 2005 (ELALOUF, Marie-Laure (dir.) et al., Écrire entre 10 et 14 ans, un corpus, des analyses, des repères pour la formation. Versailles, Sceren CRDP Académie de Versailles, 2005, 174 pages.
} 
Catherine Boré UCP-IUFM Versailles et Modyco UMR 7114 CNRS-Université Paris X Journée InterGAP «Interactions et apprentissages » 9 février 2007

collectivement; dans ce cas, les mots utilisés au cours de cette « rédaction conversationnelle ${ }^{4}$ deviennent autonymes ; dans les séances de grammaire, ils s'ajoutent aux mots métalinguistiques pris comme autonymes, qui correspondent aux notions nouvelles mises en place; cela se produit également quand la discussion orale porte sur des textes rédigés par les élèves : il s’agit alors de revenir sur du «déjà dit », ce qui autonymise automatiquement les segments écrits auxquels il est référé.

Pour sélectionner parmi tous ces aspects, on a retenu de s’intéresser :

a) à certains exemples de dénomination et de désignation que l'on a sélectionnés à partir des formules sémiotiques opposées : Y s'appelle « $\mathrm{X}$ », et : « $\mathrm{X}$ » désigne $\mathrm{Y}^{5}$, dans lesquelles « $\mathrm{X}$ » est un autonyme ${ }^{6}$. Comme on va le voir dans les exemples, la comparaison entre les deux formules fait apparaître une écrasante activité de dénomination magistrale, alors que la désignation est massivement le fait des élèves.

En outre, la dénomination, si elle entre à part à peu près égale en grammaire et en géométrie quant au nombre d'interactions orales relevées, diffère fonctionnellement dans la praxis des deux disciplines: en grammaire elle constitue pratiquement le but de la leçon quand il s'agit (par exemple) de nommer une proposition relative, dont on vient d'élucider le fonctionnement, pour la différencier d’une complétive; tandis qu'en géométrie, ne pas connaître le nom des côtés du triangle rectangle constitué par l'équerre n’empêche pas la construction de deux droites perpendiculaires.

b) au marquage de l'interaction élèves/élèves, et élèves / maître qui intervient presque uniquement dans le cadre du métalangage naturel, avec des formulations auto- ou hétéroconstituées, comme on le verra.

\footnotetext{
${ }^{4}$ APOTHELOZ, Denis, « Usages de l'autonymie dans des rédactions conversationnelles » in LOPEZ-MUNOZ, Juan-Manuel, MARNETTE, Sophie, ROSIER, Laurence, Le discours rapporté dans tous ses états, Paris, L'Harmattan, 2004, pp.335-352.

${ }^{5}$ Ces formulations, volontairement simplifiées dans cette introduction, sont empruntées à Josette Rey-Debove, op.cit.

${ }^{6}$ Pour touts ces analyses, je me réfère à l'ouvrage fondateur de REY-DEBOVE et, ponctuellement, aux analyses de J. AUTHIER-REVUZ dans AUTHIER-REVUZ, Jacqueline, DOURY, Marianne, REBOUL-TOURÉ, Sandrine, (eds), Parler des mots, le fait autonymique en discours, Paris, Presses de la Sorbonne Nouvelle, 2003, pp. 68-97.

Dans tous les cas, on définit l'autonymie comme la propriété du langage à parler de lui-même, à parler des mots qu'il utilise, à se citer lui-même, et le mot autonyme comme «l'homonyme du mot qu'il désigne ;ainsi /table /(= le mot »table ») n’est pas table (= le meuble) ».(Rey-Debove, op.cit. p.28-29).
} 
Catherine Boré UCP-IUFM Versailles et Modyco UMR 7114 CNRS-Université Paris X Journée InterGAP «Interactions et apprentissages » 9 février 2007

Présentation rapide du contexte :

Les observations s'appuient sur un corpus recueilli en classe primaire (CM2) ${ }^{7}$, et constitué par la transcription de deux séquences d'apprentissage menées par le même enseignant en géométrie et grammaire; dans les deux cas, l'enseignant procède en alternant le dialogue à visée métacognitive, et l'écrit de synthèse. En géométrie, l'objet de la séquence consiste à être capable d'exposer par écrit comment construire deux droites perpendiculaires à l'aide de la règle et de l'équerre. En grammaire, l’objet de la séquence consiste à faire identifier les propositions subordonnées relatives et les propositions subordonnées complétives et faire ensuite rédiger en groupes une synthèse écrite attestant du savoir acquis par les élèves. Dans les deux cas, il y a eu une leçon préalable, des manipulations ou des exercices, un retour oral explicité sur la leçon, et trois séances d’écriture.

\section{DÉNOMMER}

\subsection{Formule de dénomination}

On peut partir de la formule-type de la dénomination rappelée par Rey-Debove (1978:185 sq.).

$\mathrm{Y}$ s’appelle « $\mathrm{X}$ » (où « $\mathrm{X}$ » est l'autonyme).

a pour nom

se dit

se nomme

parfois :

ce $\mathrm{Y}$ s’appelle un « $\mathrm{X}$ »

où X est le nom d'une classe incluse dans celle d'un Y (ex : cette phrase est une proposition, ce mot est un nom etc. qui équivalent à : cette phrase se nomme "proposition "; ce mot se nomme « nom »),

et rechercher dans les interactions les occurrences exactes ou approchantes de ces formules.

\footnotetext{
${ }^{7}$ Classe de Loïc Martin à Argenteuil. Recherche effectuée dans le cadre de l’IUFM de Versailles, qui a donné matière à une publication : ELALOUF, Marie-Laure \& al. (2005) op.cit.
} 
Catherine Boré UCP-IUFM Versailles et Modyco UMR 7114 CNRS-Université Paris X Journée InterGAP "Interactions et apprentissages » 9 février 2007

\subsection{Exemples de dénomination}

On trouve des exemples-types de dénomination plus fréquemment en grammaire qu'en géométrie.

La dénomination y a pour particularité de s'effectuer à l'aide d’un métalangage supposé acquis, au moyen des mots métalinguistiques sujet, verbe, phrase, proposition etc.

On constate, dans l’exemple suivant, le caractère nécessairement unilatéral de l'échange interactif, l’enseignant détenant seul l'étiquette par laquelle il dénomme le référent, à savoir les exemples de phrases au tableau.

\section{EX1 (Grammai re, séance 2)}

223 M. donc ça veut dire que celles-ci où je vais nager / qui me plaît beaucoup / dont tu es le héros / sont des propositions <

224 les élèves : subordonnées

225 M. : ah on avance/ sont des propositions subordonnées / eh oui/ sauf qu'elles ont un nom+++[silence] puisque les autres s'appelaient des propositions subordonnées COMPLETIVES/ celles-là elles s'appellent des propositions subordonnées/mais pas complétives puisqu'on l'a déjà utilisé++ alors ça vous pouvez pas le savoir puisqu'on / ça peut pas s'inventer/ ça s'appelle des propositions subordonnées RELATIVES

Parfois, une question de dénomination n'est pas comprise, parce que l'autonyme n'est pas identifié comme tel.

\section{EX2 (Grammaire, séance 2)}

63 M.qu'est-ce que c'est piscine

$64 \mathrm{E}$ : un endroit où $\mathrm{y}$ a de l'eau

[Rires]

65 M. : d'accord oui mais en grammaire<

66 des élèves : c'est un groupe nominal+c'est le sujet

67 M. : c'est un groupe nominal mais sinon en lui-même c'est <

68 les élèves : un nom

L'élève propose, en 64

a) une désignation mondaine, en dépit du fait que l'autonyme " piscine » est classiquement signalé par l'absence du déterminant.

b) sous forme de définition de chose.

Or l'enseignant attend une dénomination métalinguistique, qui arrive en 68, et qu'on pourrait retraduire par : « le mot piscine s'appelle (ou est) un nom ».

On constate alors que la formule sémiotique de départ a subi une différence. : non plus : $Y$ s’appelle « $X$ », mais bien : « $X$ » est un $Y$, qui diffère de la formulation avec « être » 
Catherine Boré UCP-IUFM Versailles et Modyco UMR 7114 CNRS-Université Paris X Journée InterGAP "Interactions et apprentissages » 9 février 2007

présentée plus haut comme une variante de dénomination. En réalité, la formule syntaxique inverse l'ordre de la prédication (autonyme en tête) ce qui a peut-être pour effet de provoquer des réponses hasardeuses.

Le fait que l'autonyme fasse problème se rencontre en classe de grammaire plus souvent qu’en géométrie, où la dénomination concerne l’identification du référent comme dans cet exemple :

\section{EX3 (Géométrie, séance 3)}

(33) M. : qu'est-ce qu'on a d'autre encore < qu'est-ce que c'est que cet angle +là ?

(34) E.: il y a trois arêtes++

(35) M.: trois arêtes <++ hum pas tout à fait +on est pas dans un volume là +++ Salem <

(36) Salem : c'est un angle droit

En 33 l'enseignant réfère à la figure mondaine tracée au tableau dont il attend qu'elle soit dénommée ; l’intervention 34 est une description (inadéquate) du triangle rectangle au tableau et la réponse 36 est ambiguë : dénomination mondaine de la chose « angle droit », ou nom de la notion selon la formule « Y a pour nom « angle droit »?

Ces exemples montrent, en tout cas, que lorsqu'il s'agit d'une dénomination de "vérification » ou d' " étiquetage », que ce soit en grammaire ou en géométrie, l'interaction est très pauvre entre les élèves et l'enseignant ; ce dernier est souvent seul à détenir le mot (métalinguistique ou spécialisé) qui correspond à une notion, ou à sa représentation quand il s'agit de figures. Il peut d'ailleurs choisir d'introduire le mot correspondant à la notion au début ou à la fin de la leçon, et c'est ce qui détermine le style didactique de l'apprentissage.

Les phrases-exemples, occurrences en grammaire, posent un problème particulier car ce sont les autonymes des mots ou groupes de mots constitutifs de ces phrases qui réfèrent à la notion qu'il s’agit de dénommer (cf exemple 2). En grammaire, l'enseignant est donc confronté à la nécessité d'utiliser comme autonymes à la fois des mots mondains, issus des phrasesoccurrences, qu'il autonymise (cf «piscine »), et les nouveaux mots métalinguistiques qu'il introduit (exemple 1 : les RELATIVES), alors que par ailleurs des mots métalinguistiques sont utilisés de façon non autonymique (ex : quel est le groupe sujet de la phrase? où se trouve la proposition relative? etc.). 
Catherine Boré UCP-IUFM Versailles et Modyco UMR 7114 CNRS-Université Paris X Journée InterGAP "Interactions et apprentissages » 9 février 2007

A ces difficultés s’ajoute le fait que la dénomination peut ne pas porter sur le métalangage technique de grammaire ou de géométrie, mais sur le métalangage didactique à l'aide duquel s'effectue la leçon (voir l'exemple de « consigne » plus bas).

On s’aperçoit alors que l’interaction élèves /élèves ou maître/élèves est beaucoup plus développée car la glose y est aussi plus fréquente. Les élèves sont collectivement engagés dans la tâche de dénomination, et les antagonismes et coopérations apparaissent plus facilement, parce que, généralement, il s'agit d'attribuer un nom moins technique à un référent qui est aussi plus flou. Les élèves se sentent ainsi autorisés à prendre la parole et à confronter leurs positions, car la compétence sollicitée ne repose pas sur le savoir ou sur une compétence cognitive spécifique. Je prends ci-dessous quelques exemples.

\subsection{Dénomination et métalangage didactique}

\section{EX4 (Géométrie, séance 2)}

Les élèves comparent les textes 1 à 3 ci-dessous, qui ont été rédigés par trois élèves de la classe. La discussion collective porte sur la partie soulignée du texte 3.

\section{TEXTE $\mathrm{N}^{\circ} 1$}

Prends ta règle et trace une droite D1 place un point A puis prends ton équerre et mets le côté de l'angle droit et trace un trait. Prends ta règle et fais pareil de l'autre côté et vérifie si tes droites sont perpendiculaires.

\section{TEXTE $\mathrm{N}^{\circ} 2$}

J'ai tracé une droite D1 sur cette droite j’ai placé le point A.

Sur ce point A j'ai placé le coin de l'équerre qui sert aux angles droits puis j’ai tracé l'angle droit.

Alors j'ai fait la même chose de l'autre côté de la droite D1.

Et ça m’a donné 2 droites perpendiculaires.

TEXTE $\mathrm{N}^{\circ} 3$

Pour faire 2 droites perpendiculaires avec l'aide de la règle et de l'équerre

On trace une droite d1 à l'aide de la règle. Ensuite, on prend l'équerre et on la pose au contact de la droite d1.On prend le plus petit côté de l'équerre (celui qui forme l'angle droit) on le pose sur la droite. Après avoir posé son équerre sur la doite $\mathrm{d} 1$ tu traces une droite. Ensuite tu la prolonges à l'aide de ta règle. Après, tu vérifies que tes 2 droites sont perpendiculaires.

77 M. : alors comment vous appelleriez cette $1^{\text {re }}$ partie qui est soulignée là <

78 E1: c'est comme une consigne

$79 \mathrm{E} 2$

le titre

80 M. : (...) est-ce que vous pensez que ce qui est souligné là + est + on doit appeler ça une consigne/ on doit appeler ça un titre / est-ce qu'on doit appeler ça une consigne/ ou est-ce qu'on doit appeler ça autrement $<$ un à la fois / Clyd /

81Clyd : là ils nous disent avec quoi il faut faire +++ euh +parce qu'une consigne par exemple que ça peut être un ordre / par exemple que ça peut être trace +++ 
Catherine Boré UCP-IUFM Versailles et Modyco UMR 7114 CNRS-Université Paris X Journée InterGAP «Interactions et apprentissages » 9 février 2007

Le sens de « appeler $\mathrm{Y}$ « $\mathrm{X}$ » », sans cesser d'être métalinguistique, glisse vers la description et les élèves vont quitter le terrain strict de la dénomination pour alterner dénomination et désignation. Ils le font en cherchant d'abord à donner un nom à la phrase référencée comme « soulignée ». Mais ici, « appeler » cette phrase n’est pas lui donner un nom métalinguistique grammatical; il s’agit plutôt, par le jeu de prédications d’équivalences substituées les unes aux autres comme :

-c’est comme une consigne

-est-ce qu'on doit appeler ça une consigne

-une consigne par exemple que ça peut être un ordre

d'attribuer un nom métalinguistique didactique correspondant à la chose plutôt qu'au mot « consigne ». Les gloses successives aboutissent alors à la désignation de ce qu'est la chose " consigne ", à savoir ce qui permet de faire un exercice selon la reformulation magistrale finale :

\section{EX5 (Géométrie, séance 2)}

141 M. : j’ai demandé ce que c'était qu'une consigne + Affid <

142 Affid: une consigne c'est grâce à elle que on peut réaliser l'exercice + par exemple

143 M. d'accord / la consigne c'est ce qui permet de réaliser l'exercice

La désignation est donc une opération constante à l'intérieur même de la tâche de dénomination, et elle est aussi celle qui se prête le mieux à l’interaction.

\section{DÉSIGNER}

2.1. formule(s) sémiotique(s) de désignation ${ }^{8}$

« $\mathrm{X}$ » (= mot métalinguistique utilisé comme autonyme) désigne $\mathrm{Y}$ (où $\mathrm{Y}$ est une chose)

$$
\left\{\begin{array}{l}
\text { veut dire } \\
\text { signifie } « Y »{ }^{9}
\end{array}\right.
$$

Dans ce cas de figure, le plus répandu, que ce soit en géométrie ou en grammaire, la définition va, le plus souvent, laisser place à la description.

La périphrase, désignant une chose ou un signe, est introduite par l'effet d’un détour. Ainsi dans l'échange suivant :

\footnotetext{
${ }^{8}$ Josette REY-DEBOVE, op.cit., p.189 et sq.

${ }^{9}$ On remarque que, dans ce cas, l'objet du verbe « veut dire » ou « signifie » est un autonyme.
} 
Catherine Boré UCP-IUFM Versailles et Modyco UMR 7114 CNRS-Université Paris X Journée InterGAP «Interactions et apprentissages » 9 février 2007

\section{EX6 (Grammaire, séance 2)}

117 M. : d'accord + donc pour vous + c'est parce qu'elle est après qu'elle est subordonnée + qu'est-ce que ça veut dire principale [Silence de quelques secondes]

118 Un élève : c'est euh le verbe qui construit la phrase

119 M. : c'est le verbe qui construit la phrase < [Silence de quelques secondes] qu'est-ce que c'est < le principal dans un collège /

120 Un élève : le plus important

121 M. : il a pas levé le doigt alors +++ Clyd /

122 Clyd [hésitation]: le plus important +++

123 M. : Elodie

124 Elodie : celui qui commande le collège <

125 M. : celui qui commande le collège / alors y aurait pas quelque chose / là dans la proposition principale <

126 Un élève : il dirige tout

127 .M.: il dirige tout / ben maintenant on va revenir à la grammaire

a) on passe d'un mot autonyme métalinguistique (" principale ») à un mot autonyme mondain (« le principal »).

b) ce dernier mot est défini par une série d’équivalences :

-le plus important

-celui qui commande le collège

- il dirige tout

qui sont des éléments de signifié du mot mondain fournis dans l'interaction par accumulation de traits descriptifs.

Pour l'enseignant, cette définition sert de prétexte à l'introduction d'une motivation linguistique justifiant le sens métalinguistique de « principale ».

Ce procédé se retrouve en géométrie de façon plus subtile car les bornes séparant le métadiscours spécialisé du métadiscours mondain sont moins tranchées :

\section{EX7 (Géométrie, séance 3)}

75 M. : qui c’est qui serait OPPOSÉ à toi / heu [hésitation] Elodie dans la classe < 76 Elodie : Nassera qu- //

77 M.[interrompant] : Nassera +++ d'accord / opposé ça veut dire [silence] qu'est-ce que ça veut dire opposé < 78 plusieurs E :[Brouhaha] : éloigné / distancié>

79 M. : éloigné + ici +ici en géométrie / ça veut dire éloigné de l’angle droit / opposé + c'est qui n’est pas près de l'angle droit / on est bien d'accord/ celui là ne touche pas l'angle droit / alors que les deux côtés adjacents / touchent ici l'angle droit +++ donc on va repérer sur l'équerre

Dans l'exemple de la séance en géométrie, le détour par un mot mondain se fait de façon plus ambiguë qu'en grammaire : en 75, l’appui intonatif sur « opposé » signale de la part de 
Catherine Boré UCP-IUFM Versailles et Modyco UMR 7114 CNRS-Université Paris X Journée InterGAP «Interactions et apprentissages » 9 février 2007

l'enseignant une question en modalisation autonymique ${ }^{10}$, dont la réponse est nécessairement «mondaine». Cependant, en reposant la question de désignation de façon explicitement autonymique en 77 , le maître déclenche chez les élèves un paradigme d'équivalences strictement autonymiques (« opposé, éloigné, distancié») avant d'en arriver à la description («celui-là ne touche pas l'angle droit»), prise dans le discours et dépourvue de toute autonymie. La suite du travail, annoncée dans le dernier segment de 79 (« donc on va repérer sur l'équerre ») montre que le travail de définition se poursuit par la référenciation, non plus sur la figure représentée (un triangle rectangle construit au tableau) mais sur l'objet mondain qu'est l'équerre : il y a sans cesse passage de la désignation du type (« un côté opposé c’est Y) à l’occurrence (où se trouve le côté opposé sur tel triangle rectangle représenté au tableau, ou sur cette équerre ?). Encore que dans le cas du triangle rectangle représenté au tableau, on puisse douter que ce qui doit être désigné comme « côté opposé » par l'élève soit vraiment une occurrence de côté opposé : le type est visé à travers l'occurrence (une représentation d'objet).

Ainsi, dans les exemples cités, voit-on s'exercer un type de coopération maître /élèves qui entre dans un modèle d'interaction langagière reconnaissable : la matériel langagier initié par l'enseignant est affecté des boucles et de gloses commentatives laissées aux élèves, pas toujours prévisibles mais fortement cadrées par lui ; l'interaction inter-élèves est pauvre alors que, sur le plan linguistique, les opérations réflexives sur la langue sont riches et complexes et manifestent, de la part de élèves, une forte attention à la circulation du discours.

Cependant ce type de rapport langagier, dans lequel les deux opérations de dénomination et de désignation se croisent et s'entrecroisent, représente une petite partie de l'activité cognitivolangagière mettant en jeu du « meta ».

Une situation didactique beaucoup moins connue, en raison de son « style » langagier plus touffu, la « rédaction conversationnelle », accompagne le déroulement de toute leçon mettant en jeu de la dénomination/désignation. C’est à travers elle que l'on repère le mieux la richesse du fonctionnement métalangagier comme moteur des interactions.

\footnotetext{
${ }^{10}$ Selon la terminologie de Jacqueline AUTHIER -REVUZ.

Pour le dire vite, dans l'exemple 75, le mot « opposé » est pris à la fois en usage (il a son sens mondain) et en mention, ce que dénote l'accentuation du mot sur lequel l'attention est attirée.
} 
Catherine Boré UCP-IUFM Versailles et Modyco UMR 7114 CNRS-Université Paris X Journée InterGAP «Interactions et apprentissages » 9 février 2007

Bien que ce type de « rédaction conversationnelle » intervienne autant pour dénommer que pour désigner, c’est dans les activités de «désignation » que les exemples sont les plus nombreux.

2.2. L’autonymie dans les rédactions conversationnelles

Denis Apotheloz ${ }^{11}$ a présenté plusieurs exemples de rédactions conversationnelles comportant deux fonctionnements métalangagiers dominants :

- les énonciations qui réfèrent à un élément d’une formulation antérieurement proposée pour la commenter, l’évaluer ou le rejeter.

Elles comportent du lexique métalinguistique ou apparenté.

- la formulation du texte (du discours) même en cours de formation.

Dans le corpus de séances en classe ici analysé, on trouve des exemples de ces deux types de « retour sur » qui comportent du définitionnel.

Le point important est que les co-énonciateurs et/ou co-rédacteurs interagissent par le moyen des formulations qu'ils proposent pour le texte en construction ou pour le discours en train de s'élaborer. En effet, les élèves -surtout dans le cas très modeste des définitions- sont amenés à co-construire un métadiscours le plus souvent avec l’aide de l'enseignant.

Apotheloz propose une petite typologie qui repose sur l'opposition :

répéter/ ne pas répéter ( $\mathrm{R}$ dans notre schématisation) en aval contexte droit

VS

répéter/ ne pas répéter en amont contexte gauche.

Selon cet auteur, le premier cas est plus significatif que le second, car il marque un désaccord.

On en va voir ci-dessous dans l'exemple 8, lignes 150-151, une illustration, Clyd substituant « qu’est-ce qu’il faut faire » à « comment faire « énoncé par Kevin

2.2.1. marquage des interactions élèves /maître

\section{EX8 (Géométrie, séance 2)}

149 M. : bon alors dites-moi ce que c'est exactement qu'une consigne++ comme ça après on sera d'accord pour savoir si c'est une consigne ou pas il faut qu'on ait un critère de choix/ Kévin <

150 Kevin : c'est on explique comment fai- +++ euh [hésitation]

\footnotetext{
${ }^{9}$ APOTHELOZ, Denis, 2004, op.cit.
} 
Catherine Boré UCP-IUFM Versailles et Modyco UMR 7114 CNRS-Université Paris X Journée InterGAP « Interactions et apprentissages » 9 février 2007

151 Clyd [soufflant]: QU'EST-CE qu'il faut faire

152 Kevin : [hésitant] on donne quelque chose qu'on + justement on écrit et pour que euh comment comment il parle $<$

153 M. : est-ce que c'est tout à fait ça une consigne <

154 Kévin : [hésitant] c'est pour expliquer comment comment on va faire

155 Clyd : qu'est-ce qu'il faut faire dans l'exercice

Si l'on part de la question du maître (désignation), on va trouver successivement :

$\mathrm{R}^{12} 1$ (150): glose paraphrastique ouverte à droite

R2 (151): reprend fin R1 avec ajout en amont (à gauche)

R1 (152): nouvelle glose

$M$ (153): réorientation désignation

$\mathrm{R} 1(154)$ : répétition R1 avec variante de R1 (ajout à droite)

R2 (155): répétition de R2 + ajout à droite

Ce schéma permet de dégager une structure conversationnelle dans laquelle la coopération élèves/maître apparaît beaucoup plus complexe, selon une logique tout à la fois d'hétéro- et d'auto-reformulation. L’observation de variantes avec plusieurs élèves montre comment le texte oral devient progressivement de l'écrit :

\section{EX9 (Géométrie, séance 3)}

54 M. : alors qu'est-ce que ça va pouvoir ++ qu'est-ce ça pourrait vouloir dire que adjacent $<$ ça pourrait bien vouloir dire que adjacent $<$

55 Walid: ça complète

56 M.: non / on va essayer de réfléchir

$57 \mathrm{E} 2$ : ça complète l'angle droit

58 M. : complète < / pas tout à fait complète / attends ++je voudrais /oui<

59 E 3: qui font l'angle droit

$60 \mathrm{M}$. :alors/ qui font l'angle droit [silence]

$61 \mathrm{E} 4$ : qui forment l'angle droit

$62 \mathrm{M}$. : qui forment l'angle droit / très bien

63 M. : donc ici les côtés adjacents / les côtés adjacents / ça signifie qui forment [silence, écriture au tableau] qui font si vous voulez +++ [écriture au tableau] qui touchent l'angle droit > [écriture au tableau simultanée]

\section{Schéma}

La question de désignation du maître ouvre :

R1 (55): glose paraphrastique ouverte à droite

$\mathrm{M}$ (56) :réorientation

R2 (57)répétition R1+ ajout à droite

$\mathrm{M}$ (58)réorientation

R3 (59)substitution

$\mathrm{M}(60)$ répétition R3confirmation

R4(61) substitution par synonymie

M (62)répétition R4 confirmation

M (63)répétition R4confirmation +R3+ ajout-modification à droite

\footnotetext{
${ }^{12} \mathrm{R}=$ répéter
} 
Catherine Boré UCP-IUFM Versailles et Modyco UMR 7114 CNRS-Université Paris X Journée InterGAP « Interactions et apprentissages » 9 février 2007

L'exemple 9 marque de façon particulièrement frappante l'importance de l'autonymie dans la recherche collective d'une désignation. La collaboration des élèves et de l'enseignant (M.) toute limitée au mot qu'elle soit - n’est cependant pas de pur verbalisme : les équivalences successives font /forment/touchent [l'angle droit] participent à l'élaboration d'une notion sans la réduire à son seul signifié.

\subsection{2. marquage des interactions élèves /élèves :}

\section{EX10 (Grammaire, séance 2) ${ }^{13}$}

348 Sandra : dans chaque phrase il y a deux verbes et deux sujets

349 M. [écrivant au tableau.]: deux verbes dans chaque phrase ++ après <

[Une élève lève le doigt] Alicia <

350 Alicia : ben presque toutes les phrases / ben y a / dans le sujet y a un sujet et un verbe

$351 \mathrm{M}$. : est-ce que vous êtes tous d'accord <

352 les élèves : oui non pas dans toutes

353 M. : Sébastien <

354 Sébastien : y en a deux / y en a deux / phrases où y a un verbe ++

355 Alicia : j'ai pas dit qu’y avait dans toutes les phrases/ j'ai dit presque toutes les phrases ++

356 M. : là y en a une sur laquelle ça marche pas / sur trois / y en a une sur laquelle ça marche pas d'accord <

alors / y a des phrases / ce qu'on peut dire c'est que dans certaines phrases +++

357 Alicia : ben pour le sujet / y a un verbe et un sujet

358 M. [écrivant] : Dans certaines phrases il y a un verbe dans le sujet

(359)Elodie : des fois y a un autre sujet dans le sujet

(360)_. M. : y a un autre sujet dans le sujet /

[écrivant] : Dans certaines phrases il y a un verbe dans le sujet et un autre sujet dans le sujet $<\mathrm{d}$ 'accord $<$

Sans entrer dans le détail de l'analyse, on voit bien ici apparaître deux formes de l'activité métalangagière :

-tout d'abord, l'utilisation explicite de l'autonymie au sens strict : l'élève (en 355) revient sur sa propre formulation de 350 en autonymisant ses propres mots («j’ai pas dit...j’ai dit presque...) afin de les opposer comme arguments à l’objection d’un autre élève.

- ensuite, comme dans l'exemple 9, l'enseignant (M) répète ou modifie les propositions divergentes du groupe-classe. Mais, à la différence des exemples précédents, l’activité

\footnotetext{
${ }^{13} \mathrm{Au}$ tableau sont affichées les phrases suivantes :

Souligne les verbes dans les phrases suivantes,encadre leur sujet:

1. Le pantalon que tu portes appartenait à ta sœur.

2. Mon père a attrapé un poisson qui pèse près d'un kilo.

3. Le film que je regarde est un western.

4. La piscine où je vais nager possède deux bassins.

5. Ma mère m'a donné un livre qui me plaît beaucoup.

6. Les livres dont tu es le héros sont passionnants.

L'enseignant cherche à montrer que les relatives sont des propositions elles-mêmes dotées d'un sujet et d'un verbe.
} 
Catherine Boré UCP-IUFM Versailles et Modyco UMR 7114 CNRS-Université Paris X Journée InterGAP «Interactions et apprentissages » 9 février 2007

métalinguistique (et plus précisément autonymique sur les mots d’autrui en 359-360) n’est pas focalisée sur une tâche de désignation. L’autonymie déployée est tâtonnante, elle prend appui sur les mots déjà dits pour édifier un discours inédit. Loin de chercher, en effet, à faire retrouver une définition toute prête à ses élèves, l'enseignant élabore avec eux et en s’appuyant sur la diversité des dires, les éléments d’une pré-notion (celle de la proposition relative).

\section{CONCLUSION}

En dépit de l’inégalité des rapports maître /élèves dans le cas de figure que j’ai privilégié celui de la classe entière-, l'activité définitoire constitue un espace privilégié pour étudier la manière dont les co-rédacteurs et co-énonciateurs, sans quitter l'énonciation autonymique, communiquent à propos du texte ou du discours en train de se faire, et évaluent les suggestions apportées par chacun.

Sur le plan linguistique, on remarque l'extrême diversité des formes de métalangage en classe, symptôme de métacognition, selon la position défendue ici.

L'autonyme s’y taille la part du lion; il entre aussi bien dans la dénomination que dans la désignation ; il est autant mondain ( « piscine », " principal de collège ») que métalinguistique (« nom », « relative », « proposition»); il est de langue («qu'est-ce que ça veut dire principale ») comme de discours (« est-ce qu'on doit appeler ça une consigne ?»); il porte sur les mots de soi «(j'ai dit presque toutes les phrases ») comme sur les mots de l'autre (E1 : « c'est un groupe nominal », M. : c'est un groupe nominal, dans lequel l'autonymisation de la phrase est implicite). L' "arrêt sur les mots » (Authier-Revuz) est le plus souvent à l'initiative de l'enseignant, mais les dix exemples d'interaction observés montrent comment l'autonymie se généralise, se complexifie et s'enrichit en passant d'un locuteur à un autre, selon un ordre de parole multidirectionnel.

Sur le plan cognitif, les seuls moyens d'étudier les effets de ce travail de socialisation sur l'acquisition résident dans le passage à l'écrit, c'est-à-dire la transposition périlleuse du dialogue en monologue, où s'observe l'intégration des traces du discours de l'autre. Ce travail, réalisé dans la classe ici décrite, relève d'une analyse de l'écrit, il entre donc dans une problématique complémentaire mais distincte de celle de la présente communication. 


\section{Bibliographie}

APOTHELOZ, Denis, « Usages de l’autonymie dans des rédactions conversationnelles » in LOPEZ-MUNOZ, Juan-Manuel, MARNETTE, Sophie, ROSIER, Laurence.Le discours rapporté dans tous ses états, Paris, L’Harmattan, 2004, pp.335-352. APOTHELOZ, Denis , « Les formulations collaboratives du texte dans une rédaction conversationnelle » in de GAULMYN, Marie-Madeleine, BOUCHARD, Robert, RABATEL, Alain, Le processus rédactionnel, écrire à plusieurs voix, Paris, L’Harmattan, 2001, pp 49-66.

AUTHIER-REVUZ, Jacqueline, Ces mots qui ne vont pas de soi. Boucles réflexives et non-coïncidences du dire (2 tomes), Paris, Larousse, 1995, 869 pages.

AUTHIER-REVUZ, Jacqueline, DOURY, Marianne, REBOUL-TOURÉ, Sandrine, (eds), Parler des mots, le fait autonymique en discours, Paris, Presses de la Sorbonne Nouvelle, 2003, 383 pages.

CHEVALIER, Jean-Claude, " La place de la définition dans La Pensée et la Langue de F.Brunot (1926). Sept remarques », in La Définition, Centre d'Etudes du Lexique, Paris, Larousse, 1990, pp. 78-83.

DELESALLE, Simone, « De la définition du nom et du verbe dans la Logique et la Grammaire de Port-Royal »,) in La Définition, Centre d'Etudes du Lexique, Paris, Larousse, 1990, pp. 72-77.

DUVAL, Robert, " Découverte de la démonstration en mathématiques » in Recherches en didactique des mathématiques, vol.20/2, 2000, Grenoble, La Pensée Sauvage, pp. 139-165.

ELALOUF, Marie-Laure (dir.) \& al., Écrire entre 10 et 14 ans, un corpus, des analyses, des repères pour la formation, Versailles, Sceren CRDP Acdémie de Versailles, 2005, 174 pages.

FRANÇOIS, Frédéric : Le discours et ses entours, essai sur l'interprétation Paris, L’Harmattan, coll. « Sémantiques », 1998, 285 pages.

GOMILA, Corinne, «Le fait autonymique dans le discours didactique: comment des enseignants et leurs élèves qui apprennent à lire parlent du langage » in AUTHIER-REVUZ, Jacqueline, DOURY, Marianne, REBOUL-TOURÉ, Sandrine, (eds), Parler des mots, le fait autonymique en discours, Paris, Presses de la Sorbonne Nouvelle, 2003, 122-136.

RABATEL, Alain. (dir.), Interactions orales en contexte didactique, Lyon, Presses universitaires de Lyon, 2004, 370 pages.

REY, Alain, « Polysémie du terme définition, in La Définition, Centre d’Etudes du Lexique, Paris, Larousse, 1990, pp.1322.

REY-DEBOVE, Josette, Le Métalangage, Paris, Le Robert, coll. « L’ordre des mots », 1978, 318 pages.

VYGOTSKI, Lev, Pensée et langage, Paris, La Dispute, 2002, 537 pages. 